\title{
Anti-nociceptive and anti-inflammatory activities of the essential oil isolated from Cupressus arizonica Greene fruits
}

\author{
Sajad Fakhri ${ }^{1, *}$, Safoora Jafarian ${ }^{2, *}$, Mohammad Bagher Majnooni ${ }^{2}$, Mohammad Hosein Farzaei ${ }^{1}$, \\ Ehsan Mohammadi-Noori ${ }^{1,3}$, and Haroon Khan ${ }^{4}$
}

'Pharmaceutical Sciences Research Center, Health Institute, Kermanshah University of Medical Sciences, Kermanshah, Iran

${ }^{2}$ Student Research Committee, Kermanshah University of Medical Sciences, Kermanshah, Iran

${ }^{3}$ Regenerative Medicine Research Center, Kermanshah University of Medical Sciences, Kermanshah, Iran

${ }^{4}$ Department of Pharmacy, Abdul Wali Khan University Mardan, Mardan, Pakistan

Received July 29, 2021

Revised September 11, 2021

Accepted September 11, 2021

Handling Editor: Jong Yeon Park

\section{Correspondence}

Sajad Fakhri

Pharmaceutical Sciences Research

Center, Health Institute, Kermanshah

University of Medical Sciences,

Kermanshah 6734667149, Iran

Tel: +98-83-34276489

Fax: +98-83-34276493

E-mail: pharmacy.sajad@yahoo.com

Haroon Khan

Department of Pharmacy, Abdul Wali

Khan University Mardan, Mardan 23200 ,

Pakistan

Tel: +92-3329123171

E-mail: hkdr2006@gmail.com

*These authors contributed equally to this work as first authors.
Background: Cupressus arizonica Greene is a coniferous tree with great importance in fragrance and pharmaceutical industries. Essential oils from C. arizonica (EC) have shown potential antioxidant, and anti-microbial activities. This study aimed at investigating the anti-nociceptive and anti-inflammatory effects/mechanisms of EC.

Methods: The EC was evaluated for anti-nociceptive and anti-inflammatory activities on male Wistar rats using a formalin test and carrageenan-induced paw edema, respectively. Also, we pre-treated some of the animals with naloxone and flumazenil in the formalin test to find out the possible contributions of opioid and benzodiazepine receptors to EC anti-nociceptive effects. Finally, gas chromatography/mass spectrometry (GC/MS) analysis was used to identify the EC's constituents.

Results: EC in intraperitoneal doses of 0.5 and $1 \mathrm{~g} / \mathrm{kg}$ significantly decrease the nociceptive responses in both early and late phases of the formalin test. From a mechanistic point of view, flumazenil administration 20 minutes before the most effective dose of EC $(1 \mathrm{~g} / \mathrm{kg})$ showed a meaningful reduction in the associated antinociceptive responses during the early and late phases of the formalin test. Naloxone also reduced the anti-nociceptive role of EC in the late phase. Furthermore, EC at the doses of $1,0.5$, and $0.25 \mathrm{~g} / \mathrm{kg}$ significantly reduced paw edema from 0.5 hours after carrageenan injection to 4 hours. GC/MS analysis showed that isolated $\mathrm{EC}$ is a monoterpene-rich oil with the major presence of $\alpha$-pinene $(71.92 \%)$, myrcene (6.37\%), $\delta$-3-carene (4.68\%), $\beta$-pinene (3.71\%), and limonene (3.34\%).

Conclusions: EC showed potent anti-nociceptive and anti-inflammatory activities with the relative involvement of opioid and benzodiazepine receptors.

Key Words: Analgesics, Opioid; Anti-Inflammatory Agents; Antioxidants; Cupressus; Gas Chromatography-Mass Spectrometry; Inflammation; Oils, Volatile; Pain Measurement; Rats, Wistar; Receptors, GABA-A.

\section{INTRODUCTION}

Pain is an anticipated result of numerous conditions such as trauma, diseases, and surgical interventions. It is a complicated experience encompassing behavioral, affective, and cognitive features associated with mental pro- (c) This is an open-access article distributed under the terms of the Creative Commons Attribution Non-Commercial License (http://creativecommons.org/licenses/by-nc/4.0/), which permits unrestricted non-commercial use, distribution, and reproduction in any medium, provided the original work is properly cited.

(c) The Korean Pain Society, 2022
Author contributions: Sajad Fakhri: Methodology; Safoora Jafarian: Investigation; Mohammad Bagher Majnooni: Investigation; Mohammad Hosein Farzaei: Supervision; Ehsan Mohammadi-Noori: Formal analysis; Haroon Khan: Supervision. 
cesses $[1,2]$. Pain can have negative effects on the quality of life and reduces daily activities. Each person feels this displeasing experience at least once in their lifetime [3]. Generally, pain can be categorized into three different types, namely nociceptive, inflammatory, and pathological pain [4]. Nociceptive and inflammatory pain are protective. Nociception occurs as a result of noxious stimuli, including chemical irritants, mechanical force, heat, and cold. Inflammatory pain is linked with infection, tissue injury, and inflammatory cell infiltration. Pathological pain is not protective, and results from dysfunctional or neuropathic pain [5]. Nociception is a process involving a series of events that transmit information from the periphery via tissue nociceptors to the central nervous system (CNS). From another point of view, pain can be divided into acute and chronic pain [6]. Chronic pain is commonly associated with feelings of sadness and depression [7]. As a critical element of pain biology, opioid receptors have long been targeted for the treatment of pain and associated disorders, and opioids are the most widely applied anti-nociceptive, hitherto [8]. In this line, gamma-aminobutyric acid receptors are another key neurotransmission system with crucial roles in the process of pain perception and its regulation [9].

Inflammation is a complex process of the body's natural response to various stimuli, including invading pathogens, toxic materials, and physical damage that result in the accumulation of blood and plasma, as well as immune cells infiltration. The inflammatory response is regarded as a protective mechanism to dispose of offending stimuli, but if not appropriately regulated, it can last for more extended periods and create chronic inflammatory responses involved in several diseases $[10,11]$. Inflammation is characterized by five clinical symptoms, namely swelling, redness, heat, loss of function, and pain. Inflammatory response is the most common cause of pain [10]. In an inflamed tissue, pain is triggered basically through the metabolism of arachidonic acid and other pain-producing substances secreted from damaged tissue. Inflammation and pain are closely interconnected via numerous mediators, especially cyclooxygenase (COX) enzymes that are implicated in the synthesis of prostaglandins (PGs), such as PGE2 and PGF2a, found in the sites of inflammation at high concentrations [12]. The released PGs contribute to pain induction in two ways, firstly by directly stimulating pain receptors and secondly via sensitizing these receptors to the action of other substances capable of producing pain, including 5-hydroxytryptamin, histamine, and bradykinin [10].

Opioids and non-steroid anti-inflammatory drugs (NSAIDs) are among the most extensively used medications to treat pain and inflammation. However, these drugs suffer from a variety of side effects [13]. For example, NSAIDs have been reported to cause cardiovascular disorders, gastrointestinal tract damage, such as ulceration as well as liver and renal failure [3]. These challenges have prompted investigators from around the world to search for alternative therapies. In this regard, herbal medicines have proven to be effective against a variety of conditions associated with pain and inflammation. In contrast to the commonly used anti-nociceptives and NSAIDs, the use of herbal preparations produce few or no side effects [10].

Different species of coniferous plants have been used in traditional medicine as medicinal plants. Cupressus arizonica Greene ( $C$. arizonica Greene) is an evergreen coniferous tree. There are several reports on the use of this plant and its preparations for different indications like varicose veins and broken capillaries, as an astringent and tonic for skin, as a sedative, as well as for immune system stimulation [14].

In the present study, we sought to explore the antinociceptive and anti-inflammatory activities of the essential oil (EO) derived from the crushed fruits of $C$. arizonica Greene (EC) using a formalin test and carrageenaninduced inflammation model, respectively. Also, the obtained EC was subjected to gas chromatography/mass spectrometry (GC/MS) to characterize its constituents.

\section{MATERIALS AND METHODS}

\section{Chemicals}

The chemicals and drugs used in this study were provided by prominent companies. Carrageenan and ketamine hydrochloride/xylazine hydrochloride solution were supplied by Sigma-Aldrich (St. Louis, MO). Formalin and ether were purchased from Dr. Mojallali Chemical Complex Co. (Tehran, Iran). Diclofenac, naloxone, and flumazenil were purchased from Jalinus Pharmaceutical Company (Tehran, Iran).

\section{Isolation of EC}

The fruit of $C$. arizonica was collected from some regions of Kermanshah Province, Iran, from April to May 2020. The fruits were further verified by herbarium experts from the Division of Botany, Department of Biology, School of Science, Razi University, Kermanshah, Iran. Crushed fruits were pulverized using a sharp blade and used for isolation of the EC. EC isolation was carried out by the hydrodistillation method (200 g of crushed fruit in $400 \mathrm{~mL}$ of distilled water using a Clevenger apparatus for 4 hours. The obtained EC was dried with anhydrous sodium sulfate 
and kept at $4^{\circ} \mathrm{C}$ until use.

\section{Animals}

In this study, we used 65 male Wistar rats weighing 220$240 \mathrm{~g}$. These rats were kept in an animal room with standard conditions, including a temperature of $22^{\circ} \mathrm{C} \pm 2{ }^{\circ} \mathrm{C}$, relative humidity of $60 \% \pm 5 \%$ with $12 \mathrm{~h} / 12 \mathrm{~h}$ light/dark cycles. The animals had access to standard rat food and water ad libitum. The animals were accustomed to the laboratory conditions for one week before starting the experiments. The experimental procedures were approved by the Committee of Animal Ethics of Kermanshah University of Medical Sciences (KUMS) in accordance with the declaration of the National Institutes of Health guide for care and use of laboratory animals (IR.KUMS.REC.1398.1107).

\section{Anti-nociceptive activity of EC}

To investigate the anti-nociceptive effect of the EC, we used the formalin test as described previously by Dubuisson and Dennis [15] in 1997. Formalin acts as a stimulant to initiate early and late phase nociceptive behaviors [3]. To this end, $2 \%$ formalin solution (in distilled water) was prepared and used to induce a biphasic nociception reaction. In this part of the study, 20 rats were randomly allocated ( $\mathrm{n}=5$ for each group) to the following groups: Group 1 , the negative control group receiving an intraperitoneal (i.p.) injection of distilled water; Group 2, the positive control group receiving an i.p. injection of $30 \mathrm{mg} / \mathrm{kg}$ diclofenac; Group 3, the treatment group receiving an i.p. injection of $1 \mathrm{~g} / \mathrm{kg}$ EC (S1) ; and Group 4, the treatment group receiving an i.p. injection of $0.5 \mathrm{~g} / \mathrm{kg}$ EC (S2). The volume of the i.p. injection was $200 \mu \mathrm{L}$, and these treatments were carried out 20 minutes before the induction of nociceptive response via formalin injection. After the pre-treatment of the rats and using an insulin syringe, $20 \mu \mathrm{L}$ of $2 \%$ formalin solution was subcutaneously injected into the sub-plantar region of the left hind paw. Upon formalin injection, to assess the nociceptive behavior, the rats were located in an observation chamber under which a mirror had been applied at a $45^{\circ}$ angle to facilitate the observation. The nociceptive response was defined as the total time of licking/chewing/jumping. These responses were recorded by blinded observers from 0 to 5 minutes post-formalin injection (known as early or neurogenic phase). This period is followed by a 10 -minutes quiescent phase with minimal nociceptive responses, after which the late phase (known as the inflammatory phase) ensues, and lasts for 15 minutes. Meticulous observations were made in the early and late phases of the formalin test [3].

\section{Involvement of opioid and benzodiazepine receptors}

In the next step of our study, we sought to partly delineate the anti-nociceptive mechanisms of EC. To this end, we pre-treated the animals with naloxone (an opioid receptor antagonist) and flumazenil (a benzodiazepine receptor antagonist) to see whether the anti-nociceptive effects of EC are mediated through the opioid and benzodiazepine receptors, respectively. To explore the contribution of opioid and benzodiazepine receptors to the anti-nociceptive activity of EC, along with the control, diclofenac (30 mg/ $\mathrm{kg})$, and S1 (1 g/kg EC) groups evaluated in the previous part of study, 20 additional rats were divided into 4 groups, namely, those receiving naloxone ( $2 \mathrm{mg} / \mathrm{kg}$, i.p.), flumaze$\operatorname{nil}(0.5 \mathrm{mg} / \mathrm{kg}$, i.p.), naloxone $+\mathrm{Sl}$, and flumazenil + S1. In both the latter groups, rats were pre-treated with naloxone and flumazenil as antagonists of opioid and benzodiazepine receptors, respectively. These pre-treatments were accomplished 20 minutes before the i.p. injection of $1 \mathrm{~g} / \mathrm{kg}$ $\mathrm{EC}$, as the most effective dose in the anti-nociception test. About 20 minutes after EC injection, the formalin test was performed according to the previous section. Other groups just received associated treatments and underwent the formalin test after 20 minutes. In all the groups, following the i.p. injection of formalin, the total times of jumping/ licking/chewing were recorded as nociceptive responses from 0 to 5 minutes (the early phase), continued for 10 minutes with minimal nociceptive responses observed (the quiescent phase), and then the late phase, which was detectable 15 minutes after formalin injection and lasted for 15 minutes. The early and late phases of the formalin test were employed to assess anti-nociceptive responses [3].

\section{Anti-inflammatory activity of EC}

To evaluate the anti-inflammatory effect of the obtained EC, we used a carrageenan-induced paw edema model [16]. In this assay, 25 rats were divided randomly into five groups $(n=5)$ : Group 1 , the negative control group which received an i.p. injection of distilled water; Group 2, the positive control group which received an i.p. injection of $30 \mathrm{mg} / \mathrm{kg}$ diclofenac; Group 3, the treatment group which received an i.p. injection of $1 \mathrm{~g} / \mathrm{kg}$ EC (S1) ; Group 4, the treatment group which received an i.p. injection of $0.5 \mathrm{~g} / \mathrm{kg}$ EC (S2) ; and Group 5, the treatment group which received an i.p. injection of $0.25 \mathrm{~g} / \mathrm{kg}$ EC (S3). These pre-treatments were done one hour before subcutaneous injection of carrageenan $(0.1 \% \mathrm{w} / \mathrm{v}$ as an aqueous solution) into the subplantar region of the left hind paw. At time intervals of 0 , $0.5,1,2$, and 4 hours post-carrageenan injection, paw vol- 

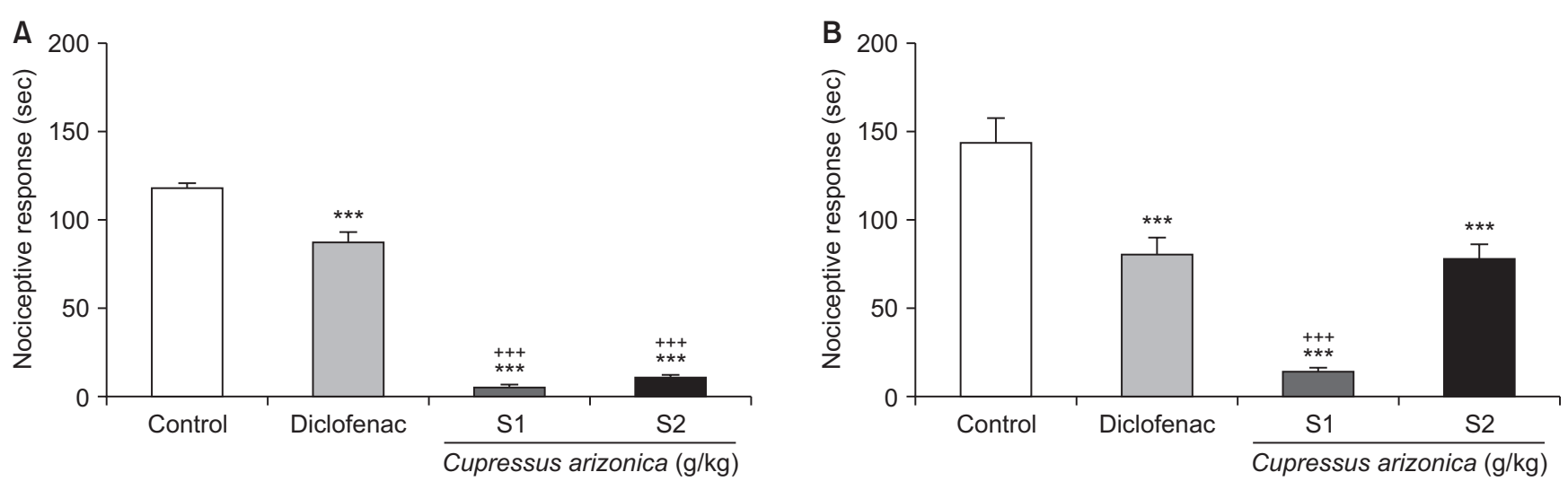

Fig. 1. Anti-nociceptive activity of Cupressus arizonica crushed fruits EC in the early (A) and late (B) phases of formalin test. The error bars indicate standard error of mean. Control: negative control group, Diclofenac: positive control group, S1: $1 \mathrm{~g} / \mathrm{kg}$ EC, S2: $0.5 \mathrm{~g} / \mathrm{kg}$ EC, EC: essential oil of $C$. arizonica. $* * * P<0.001$ vs. Control. ${ }^{+++} P<0.001$ vs. Diclofenac.

ume was measured utilizing a Plethysmometer (PM 4500; Borj Sanat Co., Tehran, Iran) [17].

\section{GC/MS analysis of EC}

We used an Agilent Technologies 7890 GC coupled to Agilent 5977A mass selective detector (MSD) (Agilent Technologies, Palo Alto, CA) with an HP5 column (length $30 \mathrm{~m}$, film thickness $0.25 \mu \mathrm{m}$, and internal diameter $0.25 \mathrm{~mm}$ ) to identify the active compounds of EC. Helium was utilized as a carrier gas, with a flow rate of $1.3 \mathrm{~mL} / \mathrm{min}$. The oven temperature was set from an initial temperature of $60^{\circ} \mathrm{C}$ programmed at a rate of $5^{\circ} \mathrm{C} / \mathrm{min}$ to $150^{\circ} \mathrm{C}$ and then heated to $250^{\circ} \mathrm{C}$ at a rate of $10^{\circ} \mathrm{C} / \mathrm{min}$. Sample injection was carried out at a split ratio of 1:100, and an electron ionization system was operated with an ionization energy of $70 \mathrm{eV}$. Finally, EC constituents were identified based on comparing their mass spectra and relative retention indices with those of standard literature compound data and the suggested library data of the GC/MS system $[18,19]$.

\section{Statistical analysis}

The obtained data were expressed as mean values \pm standard error of the mean. Data analysis was performed using GraphPad Prism software version 8 (GraphPad Software, La Jolla, CA). Also, one-way analysis of variance followed by Tukey's post hoc test were used to make comparisons between the groups. $P<0.05$ was regarded as a statistically significant difference.

\section{RESULTS}

\section{Anti-nociceptive activity of EC}

According to the results of our formalin test, EC at the dose of $1 \mathrm{~g} / \mathrm{kg}(\mathrm{S1})$, significantly reduced nociceptive responses in both phases compared to the negative control and diclofenac groups $(P<0.001)$. Besides, $0.5 \mathrm{~g} / \mathrm{kg}(\mathrm{S} 2)$ EC decreased nociceptive responses in the early and late phases $(P<0.001)$ of the formalin test compared to the negative control group. In the early phase of the experiment, the anti-nociceptive activity of $0.5 \mathrm{~g} / \mathrm{kg}$ (S2) EC at both doses was much more pronounced than the reference drug, diclofenac $(P<0.001)$. However, in the late phase, $0.5 \mathrm{~g} / \mathrm{kg}$ EC and diclofenac showed similar effects, while $1 \mathrm{~g} / \mathrm{kg}$ EC (S1) resulted in a stronger anti-nociceptive effect $(P<0.001$ compared to each group). Diclofenac, as a reference drug (positive control), also reduced nociception in both phases of formalin assay. Interestingly, in the early phase, the anti-nociceptive effects of EC at both concentrations were even significantly $(P<0.001)$ higher than that of diclofenac (Fig. 1).

\section{Involvement of opioid and benzodiazepine receptors}

Our observations revealed that in both phases of the formalin test, after antagonizing the opioid and benzodiazepine receptors using naloxone and flumazenil, the antinociceptive activity of EC ( $1 \mathrm{mg} / \mathrm{kg}$, i.p., S1) decreased, suggesting that anti-nociceptive effects of EC are to some degree mediated through these receptors. In the present report, the naloxone $+\mathrm{S} 1$, flumazenil $+\mathrm{S} 1$, and S1 groups were statistically significant with negative control and positive control groups in the early phase of formalin test $(P<0.001)$. While the naloxone $+\mathrm{S} 1$ group and S1 group 

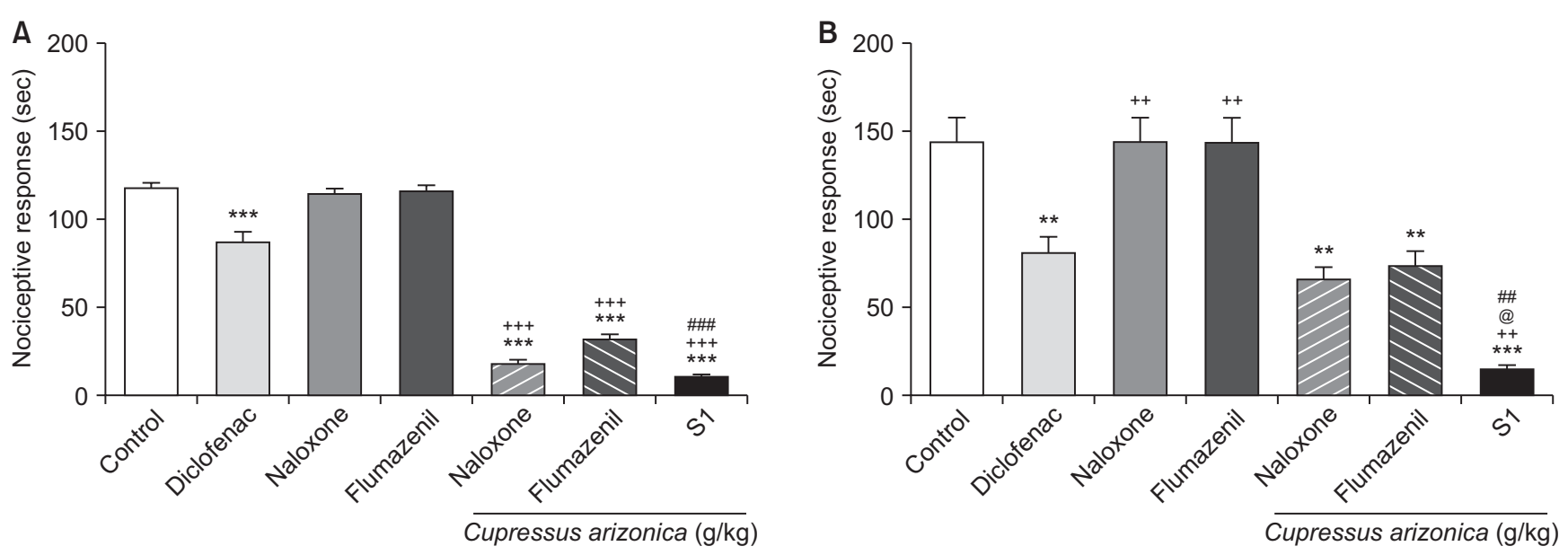

Fig. 2. Comparison of the effects of opioid (naloxone) and benzodiazepine (flumazenil) antagonists on the reduction of EC anti-nociceptive activity in the early (A) and late (B) phases of formalin test. From the left: Control: negative control group, Diclofenac: positive control group, Naloxone: the group which received naloxone and not EC, Flumazenil: the group which received flumazenil and not EC. The remaining three columns of the right-hand side underlined by "Cupressus arizonica (g/kg)" indicate the groups treated with $1 \mathrm{~g} / \mathrm{kg}$ of $C$. arizonica EC following pre-treatment with the stated antagonists (naloxone, flumazenil) or without any pre-treatment (S1). The error bars indicate standard error of mean. EC: essential oil of $C$. arizonica. $* * P<0.01$. $* * * P<$ 0.001 vs. Control. ${ }^{++} P<0.01 .{ }^{+++} P<0.001$ vs. Diclofenac. ${ }^{\#} P<0.01 .{ }^{\# \#} P<0.001$ vs. Flumazenil. ${ }^{\circledR} P<0.05$ vs. Naloxone.

showed no statistical difference in the early phase, flumazenil + S1 showed meaningful differences with the S1 group $(P<0.001)$. Consequently, flumazenil $+\mathrm{Sl}(P=0.006)$ and naloxan $+S 1(P=0.024)$ showed meaningful differences with the S1 group in the late phase of the formalin test (Fig. 2).

\section{Anti-inflammatory activity of EC}

According to the results obtained from our carrageenaninduced paw edema experiments, EC at all the doses tested $(1,0.5$, and $0.25 \mathrm{~g} / \mathrm{kg})$ resulted in significantly decreased paw edema, which indicates the anti-inflammatory activities of the EC starting from 0.5 hours post-carrageenan injection compared to the negative control group $(P<0.001)$ with no meaningful differences between those three groups. Also, diclofenac, as the reference drug, ameliorated paw edema. The anti-inflammatory effects of EC and diclofenac were increased in a time-dependent manner and peaked 4 hours after carrageenan injection. In comparison, paw edema gradually increased in the negative control group and reaching its highest level 4 hours post carrageenan injection (Fig. 3).

\section{GC/MS analysis}

As demonstrated in Table 1, GC/MS analysis of EC revealed the presence of 22 different compounds with $\alpha$-pinene, myrcene, $\delta$-3-carene, $\beta$-pinene, and limonene being the most prevalent constituents comprising respectively $71.92 \%, 6.37 \%, 4.68 \%, 3.71 \%$, and $3.34 \%$ of the total oil components (Table 1, Fig. 4).

\section{DISCUSSION}

In this study, we showed the anti-nociceptive and anti-inflammatory activities of the EO derived from the fruit of $C$. arizonica Greene, using a formalin test and carrageenaninduced inflammation model, respectively. Along with revealing its major constituents, different doses of EC significantly decreased carrageenan-induced inflammation and nociceptive responses in both early and late phases of the formalin test. The results showed that the anti-nociceptive effects of EC follow a dose-dependent pattern, evaluated by the formalin test.

The formalin test is one of the most widely used experiments to evaluate the anti-nociceptive effects of drugs. The early phase of the formalin test is related to neurogenic and non-inflammatory pain in which pain signals are transmitted through specific nervous pathways, whereas in the late phase, pain is generated as a result of formalininduced inflammatory reactions [20]. Drugs capable of acting on the nervous system affect the early phase, and anti-inflammatory agents generally ameliorate pain at the late phase of formalin assay [21]. Various anti-nociceptive agents affect the early and late phases differentially. Therefore, this test could predict the possible mechanism of action of a given anti-nociceptive/anti-inflammatory agent. Centrally-acting opioids influence both phases, while peripherally-acting anti-nociceptives like aspirin, indomethacin, and dexamethasone repress only the late phase, as this phase and the associated pain is the result of inflammatory responses [22].

Our data showed that EC is able to exert anti-nociceptive effects in both phases of the formalin test, indicating its 

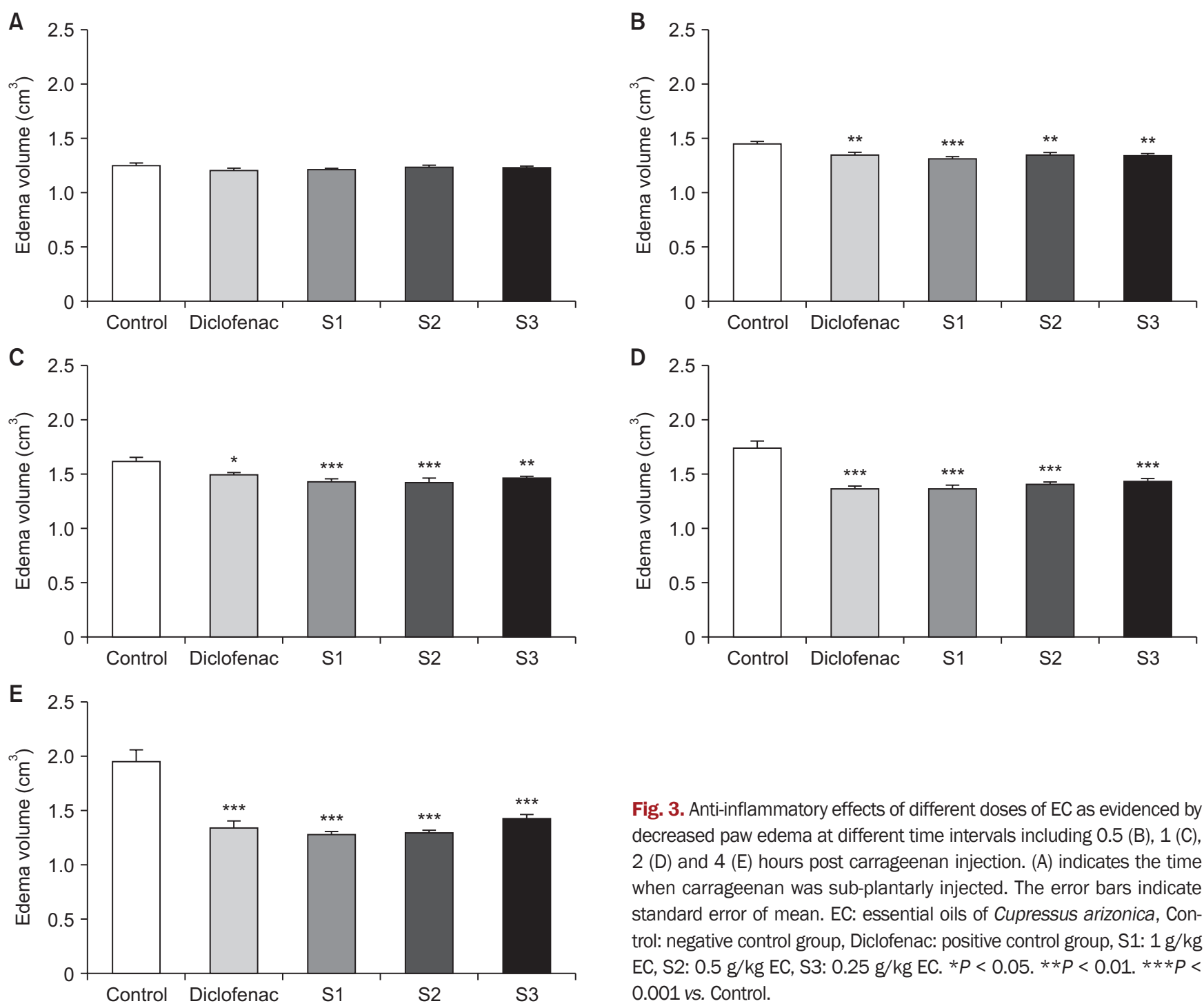

potential to act both on nociceptors (preventing the related pain signal transmission to CNS) and inflammatory reactions to alleviate pain. In the next step of our study, we tried to delineate mechanisms of EC anti-nociceptive activity. We antagonized opioid and benzodiazepine receptors using naloxone and flumazenil, respectively, and observed decreased anti-nociceptive action of EC ( $1 \mathrm{~g} /$ $\mathrm{kg}$ ) following these pre-treatments, suggesting the active involvement of these receptors in mediating the antinociceptive effects. Our observations revealed that in both phases of the formalin test, after antagonizing the opioid and benzodiazepine receptors using the mentioned antagonists, the anti-nociceptive activity of EC was decreased, suggesting that the anti-nociceptive effects of EC are to some degree mediated through these receptors (Fig. 2).

Interestingly, it appears that the relative contribution of benzodiazepine receptors to the anti-nociceptive activity of EC is greater compared to that of opioid receptors,

Fig. 3. Anti-inflammatory effects of different doses of EC as evidenced by decreased paw edema at different time intervals including 0.5 (B), 1 (C), 2 (D) and 4 (E) hours post carrageenan injection. (A) indicates the time when carrageenan was sub-plantarly injected. The error bars indicate standard error of mean. EC: essential oils of Cupressus arizonica, Control: negative control group, Diclofenac: positive control group, S1: $1 \mathrm{~g} / \mathrm{kg}$ EC, S2: 0.5 g/kg EC, S3: 0.25 g/kg EC. ${ }^{*} P<0.05$. $* * P<0.01$. $* * * P<$ 0.001 vs. Control.

as flumazenil treatment resulted in a more pronounced decrease of EC anti-nociceptive activity. Also, the antinociceptive activity of EC, as a result of flumazenil and naloxone pre-treatment, was more decreased in the late phase relative to the early phase of the formalin test, suggesting that these receptors play a more prominent role in mediating the anti-nociceptive effects of EC during the late phase in comparison to the early phase. However, the anti-nociceptive activity of EC $(1 \mathrm{~g} / \mathrm{kg})$ did not seem to be significantly affected by naloxone pre-treatment in the early phase (indicating lower dependency of EC action on such receptors) of the formalin test. In contrast, in the late phase, the difference was statistically significant (Fig. 2).

Carrageenan-induced paw edema is considered an acute inflammation event encompassing a biphasic episode, with the considerable involvement of inflammatory mediators. Thus, any medication with anti-edematous activity in this model is suggested to interfere with the production/ 
Table 1. Chemical composition of EC isolated from Cupressus arizonica crushed Greene fruits

\begin{tabular}{lrcc}
\hline \multicolumn{1}{c}{ Compound } & RI & Percentage (\%) & Identification \\
\hline Tricyclene & 926 & 0.22 & $\mathrm{MS}, \mathrm{RI}$ \\
$\alpha$-Pinene & 941 & 71.92 & $\mathrm{MS}, \mathrm{RI}$ \\
Camphene & 956 & 0.57 & $\mathrm{MS}, \mathrm{RI}$ \\
Sabinene & 976 & 0.63 & $\mathrm{MS}, \mathrm{RI}$ \\
$\beta$-Pinene & 982 & 3.71 & $\mathrm{MS}, \mathrm{RI}$ \\
Myrcene & 991 & 6.37 & $\mathrm{MS}, \mathrm{RI}$ \\
$\delta$-3-Carene & 1,013 & 4.68 & $\mathrm{MS}, \mathrm{RI}$ \\
Limonene & 1,032 & 3.34 & $\mathrm{MS}, \mathrm{RI}$ \\
(E)- $\beta$-Ocimene & 1,047 & 0.39 & $\mathrm{MS}, \mathrm{RI}$ \\
$\gamma$-Terpinene & 1,059 & 0.16 & $\mathrm{MS}, \mathrm{RI}$ \\
Terpinolene & 1,090 & 1.14 & $\mathrm{MS}, \mathrm{RI}$ \\
Linalool & 1,098 & 0.16 & $\mathrm{MS}, \mathrm{RI}$ \\
Camphor & 1,146 & 0.31 & $\mathrm{MS}, \mathrm{RI}$ \\
Umbellulone & 1,172 & 0.34 & $\mathrm{MS}, \mathrm{RI}$ \\
Terpinen-4-ol & 1,180 & 0.79 & $\mathrm{MS}, \mathrm{RI}$ \\
$\alpha$-Terpineo & 1,191 & 1.65 & $\mathrm{MS}, \mathrm{RI}$ \\
Citronellol & 1,229 & 0.69 & $\mathrm{MS}, \mathrm{RI}$ \\
Methyl carvacrol & 1,244 & 0.20 & $\mathrm{MS}, \mathrm{RI}$ \\
Bornyl acetate & 1,286 & 0.17 & $\mathrm{MS}, \mathrm{RI}$ \\
$\alpha$-Cubebene & 1,351 & 0.52 & $\mathrm{MS}, \mathrm{RI}$ \\
trans-Caryophyllene & 1,423 & 0.22 & $\mathrm{MS}, \mathrm{RI}$ \\
$\delta$-Cadinene & 1,526 & 0.22 & $\mathrm{MS}, \mathrm{RI}$ \\
\hline
\end{tabular}

EC: essential oil of $C$. arizonica, RI: retention indices, MS: mass.

release and activity of mediators of acute inflammation, including PGs and leukotrienes $[16,23]$. The obtained EC exhibited a time-dependent anti-inflammatory activity in the carrageenan-induced paw edema experiments, so that 4 hours after the carrageenan injection, maximum inhibition of edema was recorded. Notwithstanding, different doses of EC did not show significant differences in terms of their anti-edematous effects at any time interval evaluated. Also, the anti-inflammatory activity of EC (different doses) in this model was comparable to that of diclofenac. These data highlight the potential inhibitory effects of EC on acute inflammatory mediators.

Based on our GC/MS analysis, the isolated EC of western Iran contained 22 different compounds, among which $\alpha$-pinene (71.92\%), myrcene (6.37\%), $\delta$-3-carene (4.68\%), $\beta$-pinene (3.71\%), and limonene (3.34\%) were the most dominant phytochemicals. Our analysis identified terpinolene and camphene as $1.14 \%$ and $0.57 \%$ of the total oil. All of these compounds are monoterpenoids. In comparison to our study, Hassanpouraghdam [14] in 2011 evaluated the volatile fruit oil of $C$. arizonica Greene from the northwest region of Iran. The main class of components was monoterpenoids (91.9\%) followed by a minor share $(4.2 \%)$ of sesquiterpenoids, with a similar major presence of $\alpha$-pinene [14]. Emami et al. [24], in 2010, also found a predominance of $\alpha$-pinene (61.9\%), myrcene (12.6\%), limonene $(5 \%)$ and trans-sabinene hydrate $(6.8 \%)$ as the main phytochemicals of fruit's EO in Mashhad, in northwest
Iran. Upon revealing the major compounds of EC in other geographic regions, $\alpha$-pinene $(7.6 \%)$ was also found in Italy [25]. On the basis of this reported anti-oxidant activity the EO the $C$. arizonica fruit as well as there being a close interdependent relationship between inflammation and oxidative stress [26], it seemed logical for us to evaluate the anti-inflammatory effect of this oil in the present study. It should be noted that the compositional differences in the EO of $C$. arizonica fruit reported in various studies might be due to climatic and geographic factors, soil conditions, and harvest season, all causing fluctuations in the frequency of the constituents of a given oil [27]. Regarding functional studies on $C$. arizonica derived EO, several other studies have reported antibacterial and anti-fungal activities of these EO [28].

From a mechanistic point of view, anti-nociceptive and anti-inflammatory activities of the isolated EC could be attributed to its high content of monoterpene compounds. As a basic component of EC, low molecular weight monoterpenes have gained a lot of attention. These secondary plant metabolites have demonstrated numerous effects including anti-oxidant, antimicrobial, antiviral, cardioprotective, anti-tumor, anti-atherosclerotic, cytotoxic, anti-hypertensive as well as anti-inflammatory and antinociceptive activities. Many EOs that are rich in monoterpenes have elucidated pain- and inflammation-relieving effects [29].

It is obvious that EO components exert synergistic effects and considering a single compound as the major player responsible for the observed potent anti-nociceptive and anti-inflammatory activities of EO is not logical. However, we briefly delve into a number of previously published works with respect to the anti-inflammatory and antinociceptive actions of some of the major EO constituents. As a result, $\alpha$-pinene, as the major compound of $C$. arizonica fruit's EO, has been found to exert anti-nociceptive and anti-inflammatory effects [29]. In the study of Kim et al. [30] in 2015, it was found that $\alpha$-pinene acts as an antiinflammatory molecule by suppressing mitogenactivated protein kinase and nuclear factor-kappa B (NF- $\mathrm{B})$ pathways in mouse peritoneal macrophages. In another study, it was shown that $\alpha$-pinene, at non-cytotoxic doses, elicits the most potent inhibition of interleukin (IL)-1 $\beta$-induced catabolic and inflammatory pathways i.e., NF- $\kappa \mathrm{B}$ and cJun $\mathrm{N}$-terminal kinases activation and the expression of inducible nitric oxide (iNOS) and matrix metalloproteinase (MMP)-1 and MMP-13 genes [31].

Alpha-pinene has also shown anti-nociceptive effect in the tail-flick test as a commonly used model of pain in mice [32]. Orhan et al. [33] found that $\alpha$-pinene (500 $\mathrm{mg} / \mathrm{kg}$, oral gavage) has a moderate ability to decrease paw edema in a mouse model of carrageenan-induced 


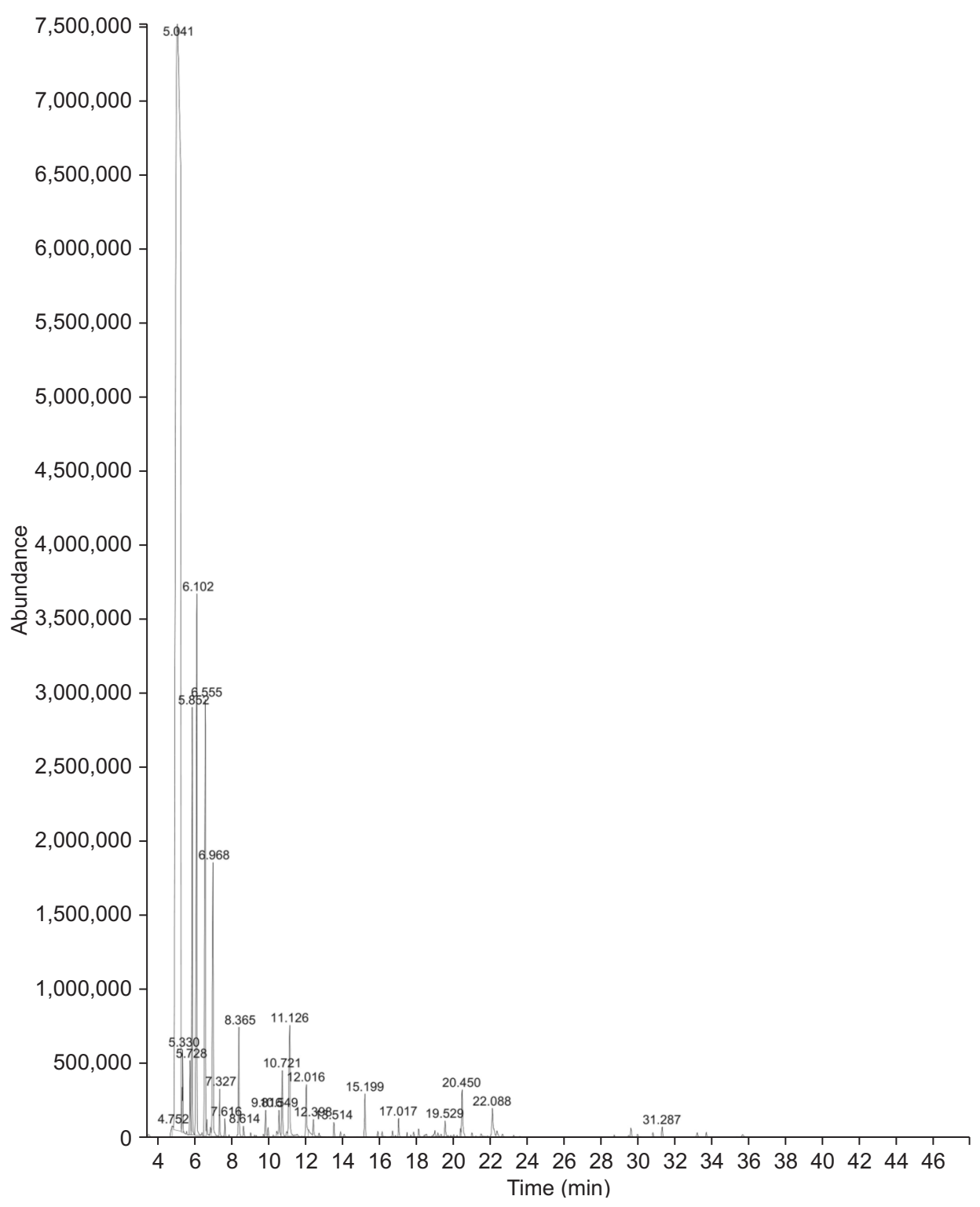

Fig. 4. GC/MS analysis of EC isolated from Cupressus arizonica Greene fruit crushed fruits. GC/MS: gas chromatography/mass spectrometry, EC: essential oil of $C$. arizonica. inflammation. Myrcene or $\beta$-myrcene, as another major monoterpene found in $C$. arizonica fruits EO, at the doses of $10-40 \mathrm{mg} / \mathrm{kg}$ (i.p. or subcutaneous) has been reported to exert anti-nociceptive effects by acting at both peripheral and central sites, and might involve the mediation of $\alpha 2$-adrenoreceptors and endogenous opioids. Additionally, myrcene hinders lipopolysaccharide (LPS)-induced inflammatory responses along with a marked inhibition of c-interferon and IL-4 production in experimental models of pleurisy. Myrcene has also exhibited a strong antinociceptive activity in an acetic acid-induced writhing test [34]. The pharmacological effects of limonene on proinflammatory cytokine production and inflammatory mediators in RAW 264.7 macrophages have been investigated by Yoon et al. [35]. It was shown that limonene inhibited LPS-induced PGE2 and NO production, as well as dosedependently decreased the expression of COX-2 and iNOS, as well as the the pro-inflammatory cytokines TNF- $\alpha$, IL6 , and IL-1 $\beta$ [35].

Taken together, our study highlighted the potent antinociceptive and anti-inflammatory activities of the EO of the fruit of $C$. arizonica. We could show that benzodiazepine and opioid receptors play critical roles in the antinociceptive effect of EC. Also, we identified 22 different compounds in the obtained EC, with $\alpha$-pinene as the most dominant constituent. Further detailed research is warranted to specify more precisely the mechanisms of the anti-nociceptive and anti-inflammatory actions of this oil.

\section{ACKNOWLEDGMENTS}

S.F. thanks Dr. Fatemeh Abbaszadeh for comments and modifications on statistical analysis. 


\section{CONFLICT OF INTEREST}

No potential conflict of interest relevant to this article was reported.

\section{FUNDING}

The authors acknowledged Pharmaceutical Sciences Research Center, Kermanshah University of Medical Sciences, for the special Grant No. 980979.

\section{ORCID}

Sajad Fakhri, https://orcid.org/0000-0001-8265-8284

Safoora Jafarian, https://orcid.org/0000-0002-7085-9748

Mohammad Bagher Majnooni, https://orcid.org/0000-0002-9470-5532

Mohammad Hosein Farzaei, https://orcid.org/0000-0001-7081-6521

Ehsan Mohammadi-Noori, https://orcid.org/0000-0002-1464-2691

Haroon Khan, https://orcid.org/0000-0002-1736-4404

\section{REFERENCES}

1. Turk DC, Meichenbaum D, Genest M. Pain and behavioral medicine: a cognitive-behavioral perspective. New York, Guilford Press. 1983.

2. Marazziti D, Mungai F, Vivarelli L, Presta S, Dell'Osso B. Pain and psychiatry: a critical analysis and pharmacological review. Clin Pract Epidemiol Ment Health 2006; 2: 31.

3. Fakhri S, Ahmadpour Y, Rezaei H, Kooshki L, Moradi SZ, Iranpanah $\mathrm{A}$, et al. The antinociceptive mechanisms of melatonin: role of L-arginine/nitric oxide/cyclic GMP/KATP channel signaling pathway. Behav Pharmacol 2020; 31: 72837.

4. Fakhri S, Abbaszadeh F, Jorjani M. On the therapeutic targets and pharmacological treatments for pain relief following spinal cord injury: a mechanistic review. Biomed Pharmacother 2021; 139: 111563.

5. Woolf CJ. What is this thing called pain? J Clin Invest 2010; 120: 3742-4.

6. Singh BM, Negi G, Bhole P, Jaiprakash M. Pain and inflammation: a review. Int J Pharm Sci Res 2012; 3: 4697-709.

7. McCleane G. Antidepressants as analgesics. CNS Drugs 2008; 22: 139-56.

8. Al-Hasani R, Bruchas MR. Molecular mechanisms of opioid receptor-dependent signaling and behavior. Anesthesiology 2011; 115: 1363-81.

9. Enna SJ, McCarson KE. The role of GABA in the mediation and perception of pain. Adv Pharmacol 2006; 54: 1-27.

10. Arome D, Sunday AI, Onalike EI, Amarachi A. Pain and inflammation: management by conventional and herbal therapy. Indian J Pain 2014; 28: 5-12.

11. Rastegari-Pouyani M, Mostafaie A, Mansouri K, MortazaviJahromi SS, Mohammadi-Motlagh HR, Mirshafiey A. Antiangiogenesis effect of $\beta$-D-mannuronic acid (M2000) as a novel NSAID with immunosuppressive properties under experimental model. Clin Exp Pharmacol Physiol 2018; 45: 370 6.

12. Deraedt R, Jouquey S, Delevallée F, Flahaut M. Release of prostaglandins $\mathrm{E}$ and $\mathrm{F}$ in an algogenic reaction and its inhibition. Eur J Pharmacol 1980; 61: 17-24.

13. Wilhelmsen M, Amirian I, Reiter RJ, Rosenberg J, Gögenur I. Analgesic effects of melatonin: a review of current evidence from experimental and clinical studies. J Pineal Res 2011; 51: 270-7.

14. Hassanpouraghdam MB. $\alpha$-Pinene- and $\beta$-myrcene-rich volatile fruit oil of Cupressus arizonica Greene from northwest Iran. Nat Prod Res 2011; 25: 634-9.

15. Dubuisson D, Dennis SG. The formalin test: a quantitative study of the analgesic effects of morphine, meperidine, and brain stem stimulation in rats and cats. Pain 1977; 4: 161-74.

16. Pandpazir M, Kiani A, Fakhri S, Mousavi Z. Anti-inflammatory effect and skin toxicity of aqueous extract of Dorema ammoniacum gum in experimental animals. Res J Pharmacogn 2018; 5: 1-8.

17. Morris CJ. Carrageenan-induced paw edema in the rat and mouse. Methods Mol Biol 2003; 225: 115-21.

18. Adams RP. Identification of essential oil components by gas chromatography/quadrupole mass spectroscopy. 3rd ed. Carol Stream, Allured Publishing Corp. 2001.

19. Babushok VI, Linstrom PJ, Zenkevich IG. Retention indices for frequently reported compounds of plant essential oils. J Phys Chem Ref Data 2011; 40: 043101.

20. Moradi N, Fakhri S, Farzaei MH, Abbaszadeh F. The anti-nociceptive activity of naringenin passes through L-arginine/ NO/cGMP/KATP channel pathway and opioid receptors. Behav Pharmacol 2021; 32: 590-8.

21. Taherian AA, Etemadi H, Sadeghi H. Assessment of aqueous extract of seed of Cuminum cyminum L. on neurogenic and inflammatory pain in mice. J. Med. Plants 2007; 6: 44-50.

22. Morteza-Semnani K, Saeedi M, Hamidian M, Vafamehr H, Dehpour AR. Anti-inflammatory, analgesic activity and acute toxicity of Glaucium grandiflorum extract. J Ethnopharmacol 2002; 80: 181-6.

23. Popović V, Petrović S, Tomić M, Stepanović-Petrović R, Micov A, Pavlović-Drobac M, et al. Antinociceptive and antiedematous activities of the essential oils of two Balkan endemic Laserpitium species. Nat Prod Commun 2014; 9: 1258.

24. Emami SA, Fakhrjafary M, Tafaghodi M, Hassanzadeh MK. Chemical composition and antioxidant activities of the essential oils of different parts of Cupressus arizonica Greene. 
J Essent Oil Res 2010; 22: 193-9.

25. Flamini G, Cioni PL, Morelli I, Bighelli A, Castola V, Casanova J. GC/MS and ${ }^{13} \mathrm{C}-\mathrm{NMR}$ integrated analyses of the essential oils from leaves, branches and female cones of Cupressus arizonica from Italy. J Essent Oil Res 2003; 15: 302-4.

26. Biswas SK. Does the interdependence between oxidative stress and inflammation explain the antioxidant paradox? Oxid Med Cell Longev 2016; 2016: 5698931.

27. Masotti V, Juteau F, Bessière JM, Viano J. Seasonal and phenological variations of the essential oil from the narrow endemic species Artemisia molinieri and its biological activities. J Agric Food Chem 2003; 51: 7115-21.

28. Chéraif I, Jannet HB, Hammami M, Khouja ML, Mighri Z. Chemical composition and antimicrobial activity of essential oils of Cupressus arizonica Greene. Biochem Syst Ecol 2007; 35: 813-20.

29. Dragomanova S, Tancheva L, Georgieva M, Klisurov R. Analgesic and anti-inflammatory activity of monoterpenoid Myrtenal in rodents. J IMAB 2019; 25: 2406-13.

30. Kim DS, Lee HJ, Jeon YD, Han YH, Kee JY, Kim HJ, et al. Alpha-pinene exhibits anti-inflammatory activity through the suppression of MAPKs and the NF- $\kappa \mathrm{B}$ pathway in mouse peritoneal macrophages. Am J Chin Med 2015; 43: 731-42.

31. Rufino AT, Ribeiro M, Judas F, Salgueiro L, Lopes MC, Cavaleiro $\mathrm{C}$, et al. Anti-inflammatory and chondroprotective activity of (+)- $\alpha$-pinene: structural and enantiomeric selectivity. J Nat Prod 2014; 77: 264-9.

32. Him A, Ozbek H, Turel I, Oner AC. Antinociceptive activity of alpha-pinene and fenchone. Pharmacologyonline 2008; 3 : 363-9.

33. Orhan I, Küpeli E, Aslan M, Kartal M, Yesilada E. Bioassayguided evaluation of anti-inflammatory and antinociceptive activities of pistachio, Pistacia vera L. J Ethnopharmacol 2006; 105: 235-40.

34. Guimarães AG, Quintans JS, Quintans LJ Jr. Monoterpenes with analgesic activity--a systematic review. Phytother Res 2013; 27: 1-15.

35. Yoon WJ, Lee NH, Hyun CG. Limonene suppresses lipopolysaccharide-induced production of nitric oxide, prostaglandin E2, and pro-inflammatory cytokines in RAW 264.7 macrophages. J Oleo Sci 2010; 59: 415-21. 